\title{
Nuevas técnicas en ablación de fibrilación auricular: tecnologías emergentes (ablación multielectrodo y balón láser)
}

\section{New techniques in atrial fibrillation ablation: emerging technologies (multielectrode ablation and laser balloon)}

\author{
Diego Ignacio Vanegas ${ }^{a, *}$, Carlos A. Rincón ${ }^{a}$ y José Luis Merino ${ }^{b}$ \\ a Unidad de Electrofisiología Cardiovascular, Hospital Militar Central, Bogotá, Colombia \\ b Unidad de Arritmias y Electrofisiología Robotizada, Hospital Universitario La Paz, Madrid, España
}

Recibido el 3 de octubre de 2016; aceptado el 6 de octubre de 2016

Disponible en Internet el 9 de noviembre de 2016

\section{Introducción}

La ablación de las venas pulmonares mediante radiofrecuencia, ha sido la técnica predominantemente utilizada en los últimos 20 años para el tratamiento de la fibrilación auricular paroxística ${ }^{1}$. El objetivo terapéutico es el aislamiento eléctrico de las venas pulmonares desde donde se originan más frecuentemente las extrasístoles desencadenantes de los episodios arrítmicos y en algunos pacientes, en donde reside el substrato arrítmico ${ }^{2,3}$. Esta estrategia consiste en aplicar lesiones de radiofrecuencia punto a punto en el ostium y/o antrum de las mismas por un tiempo y con un poder y temperatura suficientes para abatir los potenciales eléctricos generados en su vecindad ${ }^{4,5}$. Sin embargo, la eficacia de esta estrategia no es uniforme; está asociada con una tasa elevada de recurrencias con frecuente necesidad de re-intervención y riesgo de complicaciones mayores. El éxito de la técnica actual de radiofrecuencia irrigada depende de varios factores: anatomía de las venas pulmonares, experiencia del operador, magnitud del contacto y estabilidad del catéter, tipo de catéter o técnica

\footnotetext{
* Autor para correspondencia.

Correo electrónico: diegovanegascadavid@gmail.com (D.I. Vanegas).
}

utilizada, tiempo de aplicación de la lesión de radiofrecuencia, temperatura y poder alcanzados entre otros, todos ellos determinantes de una lesión transmural y consecuentemente de la eficacia del asilamiento eléctrico de las venas pulmonares. Pese a la introducción del mapeo tridimensional, el asilamiento eléctrico de las venas pulmonares consume un tiempo considerable, requiere diversas maniobras, alta exposición radiológica y gran habilidad física del operador. Las nuevas tecnologías buscan en principio reducir la complejidad, el tiempo total del procedimiento y de exposición radiológica (por ello llamados dispositivos de un solo disparo), mantener la seguridad o reducir las complicaciones y mejorar la eficacia de las lesiones transmurales lo que debe redundar en mejores resultados o eficacia del tratamiento a largo plazo ${ }^{6}$.

\section{Ablación multielectrodo}

La ablación por radiofrecuencia irrigada punto a punto es dispendiosa y puede requerir posicionamiento del catéter repetidas veces en un punto de interés interrumpiendo el flujo continuo y eficaz de energía, hecho que puede desencadenar lesiones no transmurales, edema y reconexión eléctrica de la vena. Para lidiar con estas dificultades se consideró la realización de la ablación por radiofrecuencia mediante el uso de un catéter multielectrodo con posibilidad 

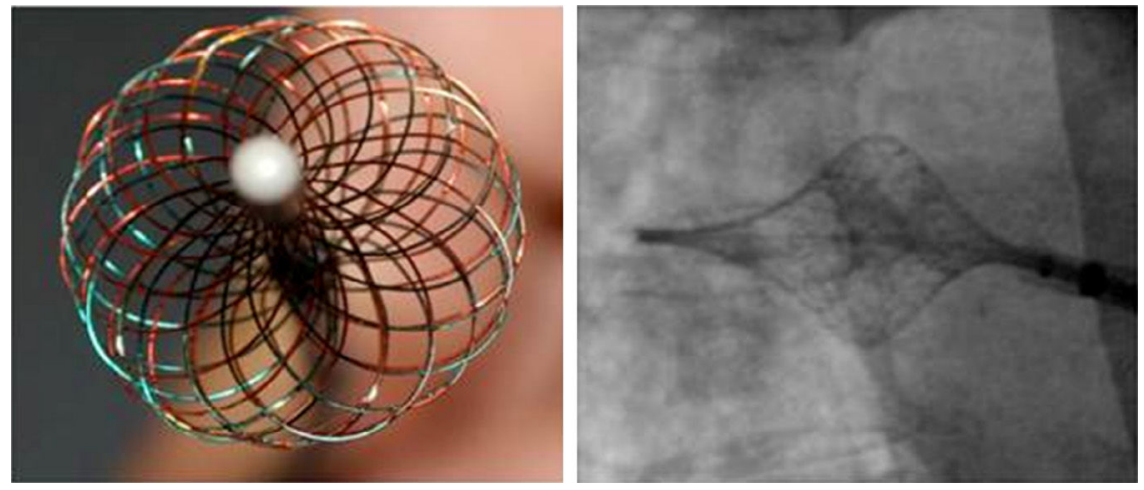

Figura 1 Catéter $\mathrm{MESH}^{\circledR}$, estructura en forma de malla o nido.

de registro de señales eléctricas de los potenciales de las venas pulmonares y simultáneamente, suministro de energía de radiofrecuencia por todos sus polos, lo que en teoría podría traducirse en la facilitación y el acortamiento del procedimiento de asilamiento eléctrico de las venas pulmonares. Uno de los primeros diseños realizados fue el catéter HDMM (High Density Mesh Maper) conocido como MESH de la firma Bard, con forma de malla flexible y adaptable al contorno de las venas pulmonares (fig. 1). Más recientemente otros diseños han cambiado a una forma de catéter multielectrodo circunferencial como el catéter de ablación de vena pulmonar PVAC (de su sigla en inglés por Pulmonary Vein Ablation Catheter, de Medtronic ${ }^{\circledR}$, Ablation Frontiers, Carlsbad, CA, USA) (fig. 2) y el catéter de ablación circular nMARQ (Biosense Webster ${ }^{\circledR}$, Diamond Bar, CA) utilizados principalmente para aquellos pacientes con fibrilación auricular paroxística.
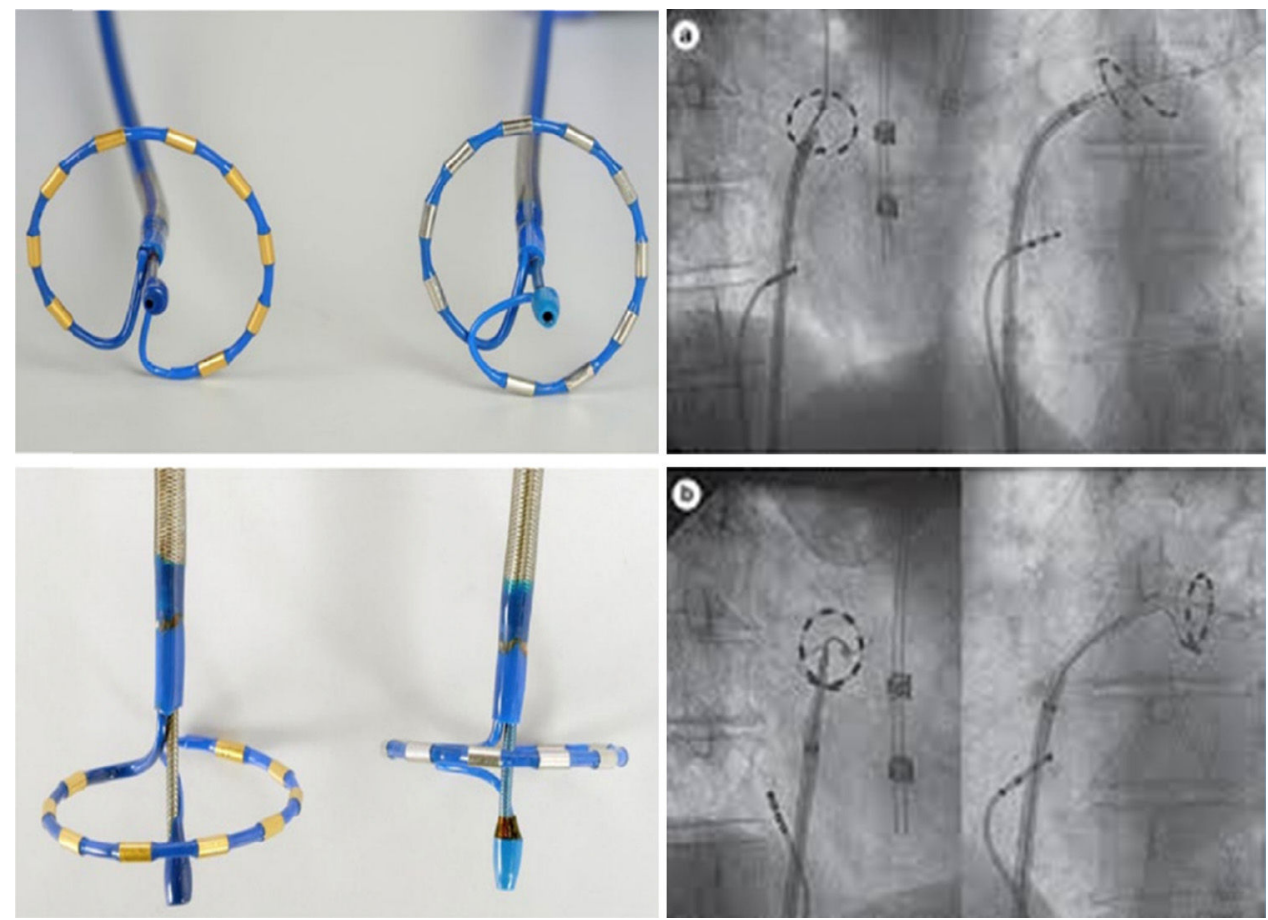

Figura 2 PVAC gold (metal oro) y modelo predecesor (metal plata) comparados en relación con su forma y grado de inclinación. Las imágenes de la derecha muestran su posicionamiento radioscópico. 
complemento con la técnica convencional de radiofrecuencia punto a punto; los autores sugirieron modificaciones en el diseño (aumento del tamaño) y la utilización de una guía "over the wire"'. El estudio europeo de evaluación clínica del mismo catéter ${ }^{8}$, mostró reconexión eléctrica frecuente de las venas tras un seguimiento de seis meses, siendo más efectivo el MESH de $35 \mathrm{~mm}$, pero con resultados finales no tan alentadores como los de la experiencia norteamericana. En general, se requirió del uso de la estrategia convencional de radiofrecuencia punto a punto por dificultad en alcanzar por completo el asilamiento eléctrico de las venas pulmonares. Más recientemente, el MESH fue comparado contra la terapia con criobalón para el tratamiento de la fibrilación auricular paroxística, confirmándose la gran dificultad en lograr un aislamiento contundente de las venas y diferencias muy significativas a favor del criobalón en el número de venas pulmonares aisladas por paciente y en el número de pacientes con todas las venas pulmonares aisladas o con bloqueo de salida? .

\section{Ablación con catéter PVAC}

El PVAC es un catéter $9 \mathrm{Fr}$, en su extremo circular, de $25 \mathrm{~mm}$ de diámetro, decapolar, con electrodos de $3 \mathrm{~mm}$ de tamaño y $3 \mathrm{~mm}$ de separación entre ellos, no irrigado, deflectable y bidireccional, que utiliza una guía 0,032 como sistema "over the wire" para su posicionamiento en la interface ostium-antro de la vena pulmonar y es insertado en la aurícula izquierda utilizando un sistema o camisa $10 \mathrm{Fr}^{10}$. Puede utilizarse para el mapeo de señales endocavitarias, principalmente potenciales de venas pulmonares, pero estas señales no son visibles al momento de aplicar radiofrecuencia. Mediante un generador específico (GENius $^{\circledR}$ radiofrecuencia generator) puede dar aplicaciones de radiofrecuencia pulsadas, simultáneamente, en modo unipolar y/o bipolar por los 10 polos o electrodos, monitorizando así la temperatura independientemente en cada canal con poder limitado entre 8 y10 watts $^{11}$. Las aplicaciones uni- y bipolares simultáneas producen una lesión más amplia y profunda que solo las unipolares; en general la lesión unipolar individual se relaciona con profundidad, mientras que la bipolar con amplitud o longitud mayores. El PVAC no es reconocido por algunos sistemas de mapeo 3-D y la aplicación de radiofrecuencia en las venas pulmonares está estandarizada a un minuto, de modo que no es posible visualizar en forma simultánea los potenciales eléctricos y en consecuencia el momento de su abolición ${ }^{12}$. Los primeros estudios de su aplicación clínica mostraron efectividad en el asilamiento eléctrico de las venas pulmonares en un $73 \%$, pero llamaron la atención acerca de la necesidad de utilizar otro catéter circular para demostrar el aislamiento e incrementar la eficacia a 93\% y cautela por la elevación crítica de la temperatura esofágica en cerca del $50 \%$ de los pacientes ${ }^{13}$. Cuando se comparó la estrategia de PVAC contra ablación por radiofrecuencia "convencional" punto a punto ${ }^{14}$ el tiempo total del procedimiento fue significativamente reducido con el uso del primero mientras que las complicaciones y la tasa de recurrencias libre de medicamentos fueron similares. Estos mismos hallazgos han sido corroborados por otros estudios aleatorizados ${ }^{15}$ que han comparado PVAC versus radiofrecuencia irrigada punto a punto sin encontrar diferencias estadísticamente significativas en la tasa de éxito a 6 meses, con recurrencias del 23 y $29 \%$ respectivamente o, del 28 y el $32 \%$ (PVAC vs. radiofrecuencia irrigado), independiente del tipo de fibrilación auricular tratada (paroxística o persistente) ${ }^{16}$; igualmente, otras series de casos no aleatorizados con PVAC mostraron resultados sólidos, con recurrencia entre 14 y $17 \%$ para ese mismo lapso de tiempo ${ }^{17,18}$. Pese a la eficacia elevada aguda en el asilamiento eléctrico de las venas pulmonares con este multielectrodo, se ha observado una caída de su efectividad al 54,2\% libre de recurrencia tras seguimiento más prolongado de 19 meses $^{19}$ y de $49 \%$ a 2 años en pacientes sin medicación antiarrítmica ${ }^{20}$. Se ha informado acerca de resultados aún más bajos tras 12 meses de seguimiento en el estudio aleatorizado AF-COR (Malmborg et al.) que comparó eficacia y seguridad de las estrategias de criobalón versus PVAC en pacientes con fibrilación auricular paroxística o persistente (46 vs. 34\%). Estos resultados contrastan con lo informado por De Greef et al. ${ }^{21}$ con una eficacia del $65 \%$ a 3 años. La utilización del PVAC para la ablación de fibrilación auricular persistente o largamente persistente ha sido probada en forma aleatoria, contra el manejo farmacológico en el estudio TTOP-AF (Tailored Treatment of Persistent Atrial Fibrillation) ${ }^{22}$; el porcentaje de pacientes libres de fibrilación auricular a un año de seguimiento fue significativamente mayor en aquellos llevados a ablación con PVAC (55,8\% vs. $26,4 \%)$; sin embargo. el porcentaje de complicaciones agudas y la tasa global de complicaciones fueron altas con relación a estudios previos y otras tecnologías $(12,3 \%$ agudas y $16 \%$ globales).

Las lesiones de radiofrecuencia producidas por el PVAC estudiadas mediante mapeo de voltaje del antrum y del ostium de las venas ${ }^{23}$, demostraron importante extensión en ambos sentidos; estos hallazgos contrastan con los encontrados después de ablación con criobalón, que focaliza las lesiones más hacia el antrum que en la unión antro-ostium ${ }^{24}$. En ese sentido y apoyando tal observación, la magnitud de la destrucción del tejido miocárdico analizada mediante la cuantificación de biomarcadores enzimáticos posteriores al procedimiento de asilamiento eléctrico de las venas pulmonares se reporta más alta con criobalón que con el PVAC, pero no por ello mejor o de mayor beneficio clínico ${ }^{25,26}$. El PVAC también ha sido recientemente comparado con el catéter $\mathrm{nMARQ}^{\circledR}$ por un estudio israeli ${ }^{12}$ encontrándose acortamiento significativo y similar en el tiempo total del procedimiento y de fluoroscopia, tasa de éxito aguda y a un año comparable para ambas tecnologías. El número de aplicaciones y el tiempo total de aplicación de energía de radiofrecuencia fue menor con el $n M A R Q$, pero dado su mayor tamaño se recomienda su uso, preferencialmente en venas pulmonares y aurícula izquierda grandes, ha llevado a sugerir un estudio detallado de la anatomía antes de la ablación para seleccionar un sistema específico. La incidencia de complicaciones para ambas tecnologías en ese estudio fue similar a la observada en otros donde se utilizó la técnica convencional de asilamiento eléctrico de las venas pulmonares con catéter irrigado punto a punto. Sin embargo, el catéter $\mathrm{nMARQ}$ circular irrigado ha sido recientemente retirado del mercado por la compañía fabricante (Biosense Webster ${ }^{\circledR}$, junio 2015) al encontrar una disfunción en los sensores de temperatura que producían una subestimación de la misma, lo que al parecer pudo asociarse a una 
incidencia mayor de fístula atrio-esofágica. En contraste, a la fecha no ha sido informado el primer caso de fístula atrio-esofágica con el uso del PVAC, aunque se han descrito diversos grados de lesión a esa estructura en el $2 \%$ de los pacientes evaluados mediante endoscopia y resonancia nuclear magnética, habiéndose observado resolución de las mismas bajo manejo conservador ${ }^{11,27}$. De otro lado, pese a que se observado I parálisis del nervio frénico secundaria a la utilización del PVAC en un solo $\operatorname{caso}^{28}$, el riesgo de dicha complicación es uno de los principales, toda vez que su captura o estimulación puede lograrse durante las maniobras de posicionamiento del multi-electrodo. Es sorprendente tal reporte aislado a pesar de las múltiples series de pacientes en diversos grupos, países y tipos de fibrilación auricular tratados. Gaita et al. ${ }^{29}$ en 2011 , informaron una tasa de isquemia cerebral asintomática posterior al uso de PVAC tan alta como de $38,9 \%$ versus $8,3 \%$ con la técnica de ablación por radiofrecuencia con catéter irrigado punto a punto. Investigaciones ulteriores sobre este tema (2013), llevaron a otros investigadores a realizar modificaciones prey transoperatorias, logrando reducir tal incidencia a cifras tan bajas como del $1,7 \%^{30}$. Los factores que al parecer redujeron la incidencia emboligéna fueron: la estrategia de no interrumpir la warfarina y manejar un tiempo activado de coagulación (ACT) mayor de 350 segundos, mantener el cuerpo del PVAC dentro de la camisa o sistema de introducción para evitar la embolia aérea, y deshabilitar el polo distal ${ }^{10}$ o proximal del catéter multielectrodo para evitar la creación de un cortocircuito bipolar entre los electrodos 1 y 10 que se ponían en contacto y que elevan la temperatura inesperadamente, siendo fuente potencial de embolia gaseosa y/sólida. Otra potencial complicación con el uso del PVAC es la estenosis pulmonar, la cual es discretamente superior a la observada con el uso de radiofrecuencia irrigada punto a punto ( 5 vs. $4 \%$ respectivamente) $)^{31}$. Así mismo, mediante tomografía computarizada se ha observado reducción significativa del área ostial de las venas pulmonares de 236 a $173 \mathrm{~mm}^{2}$ tras un año de seguimiento ${ }^{32}$. Sin embargo, esta reducción fue catalogada como leve y los pacientes cursaron asintomáticos. Los autores sugirieron la utilización de una relación en la entrega de energía bipolar/unipolar 4:1 y evitar las múltiples aplicaciones para reducir el riesgo de estenosis ${ }^{11}$. Una forma mejorada del PVAC recientemente lanzada a la arena clínica, es el PVAC con electrodos de oro o PVAC GOLD; este metal es mejor conductor que el platino de mod que permite un calentamiento más uniforme, enfriamiento más rápido y por ende un mejor control de la temperatura. Estas propiedades de electroconducción del oro, han arrojado resultados clínicos prometedores en términos de mayor eficacia en el aislamiento eléctrico de las venas pulmonares al tiempo que se ha demostrado una reducción de la incidencia de eventos embólicos isquémicos cerebrales silenciosos $^{33}$. El uso de estrategias combinadas de PVAC y ablación por radiofrecuencia irrigada punto a punto ha demostrado también mejores resultados que la ablación realizada solo con una de estas técnicas indicando que los efectos terapéuticos pueden ser favorablemente complementarios ${ }^{34}$. Finalmente, la curva de aprendizaje con el manejo de este catéter multielectrodos ha sido catalogada como corta y favorable incluso en centros de bajo volumen de aislamiento eléctrico de las venas pulmonares lo que sugiere un perfil de seguridad y eficacia balanceada ${ }^{35}$.

\section{Ablación con catéter $n M A R Q{ }^{\circledR}$}

El catéter $\mathrm{nMARQ}$ de primera generación es de 8,4 Fr, irrigado, decapolar, con polos de $3 \mathrm{~mm}$ de largo y separación entre electrodos de $4 \mathrm{~mm}$, en forma circular o de media luna en su extremo terminal, ajustable a diámetros entre 20 y $35 \mathrm{~mm}$, reconocido por el sistema de mapeo electromagnético CARTO3 gracias a tres sensores (proximal, medio y distal) localizados en los polos 1,5 y 10 del catéter, con capacidad para registrar señales endocavitarias de interés como los potenciales eléctricos de las venas pulmonares y simultáneamente suministrar energía de radiofrecuencia en modo unipolar o bipolar. A diferencia del PVAC las principales virtudes de este catéter son la visualización 3-D de los electrodos en contacto con la interface tisular gracias a la tecnología "tissue-connect", la apreciación de los potenciales eléctricos de las venas durante la radiofrecuencia y la tecnología irrigada ${ }^{12}$. En el catéter $n M A R Q$ cada polo tiene un control independiente desde el generador de radiofrecuencia (nMARQ RF Generator ${ }^{\circledR}$ ). Durante ablación el flujo es de $60 \mathrm{ml} /$ minuto con irrigación a través de 10 puertos por cada electrodo; el flujo de mantenimiento es de $4 \mathrm{ml} / \mathrm{min}$. La ablación en modo unipolar puede ser administrada a través de cada electrodo hasta un poder máximo de 25 watts; en modo bipolar se puede administrar radiofrecuencia entre polos 1-2, 2-3, 3-4, etc., con un poder hasta de 15 watts. Sin embargo, se inform \{o lesión esofágica con el uso en modo unipolar y un poder entre $20-25$ watts hasta en $50 \%$ de los pacientes, así que se sugirió un poder máximo en unipolar de 15 watts. Siguiendo estas recomendaciones, Burri et al. ${ }^{36}$ utilizaron el nMARQ en una población de 50 pacientes informando un éxito agudo en el $100 \%$ de los casos, pero una tasa de recurrencia alta de $54 \%$ tras un seguimiento de $15 \pm 4$ meses; a pesar del poder reducido se presentaron complicaciones como efusión pericárdica y parálisis del nervio frénico en dos pacientes. Estudios previos realizados por Wakili et al. ${ }^{37}$ con el uso comparativo del catéter nMARQ versus catéter convencional irrigado, mostraron que con el uso del primero, no pudieron aislarse el $17 \%$ de las venas pulmonares, principalmente las inferiores y en $65 \%$ de las venas hubo detección de reconexión eléctrica postablación en comparación con el $100 \%$ de aislamiento completo de las venas y ausencia de potenciales con el uso del segundo. El tiempo total de fluoroscopia y el tiempo del procedimiento en la aurícula izquierda fue significativamente menor para el catéter convencional irrigado. Solo el tiempo total de radiofrecuencia fue significativamente inferior con el uso del nMARQ. Un porcentaje importante de pacientes en los que se utilizó el nMARQ requirieron el uso complementario de un catéter circular de mapeo para completar el aislamiento. No hubo diferencia significativa en la tasa de recurrencia tras un seguimiento de dos años.

\section{Ablación con balón láser}

La técnica del balón láser, también conocida como sistema de ablación endoscópica (EAS Endoscopic Ablation System) o ablación con láser guiada visualmente (VGLA Visually Guided Laser Ablation) se realiza con un catéter que tiene incorporados varios elementos así: a) un balón distensible en su extremo distal, b) un endoscopio miniaturizado $2 \mathrm{Fr}, \mathrm{c}$ ) una 

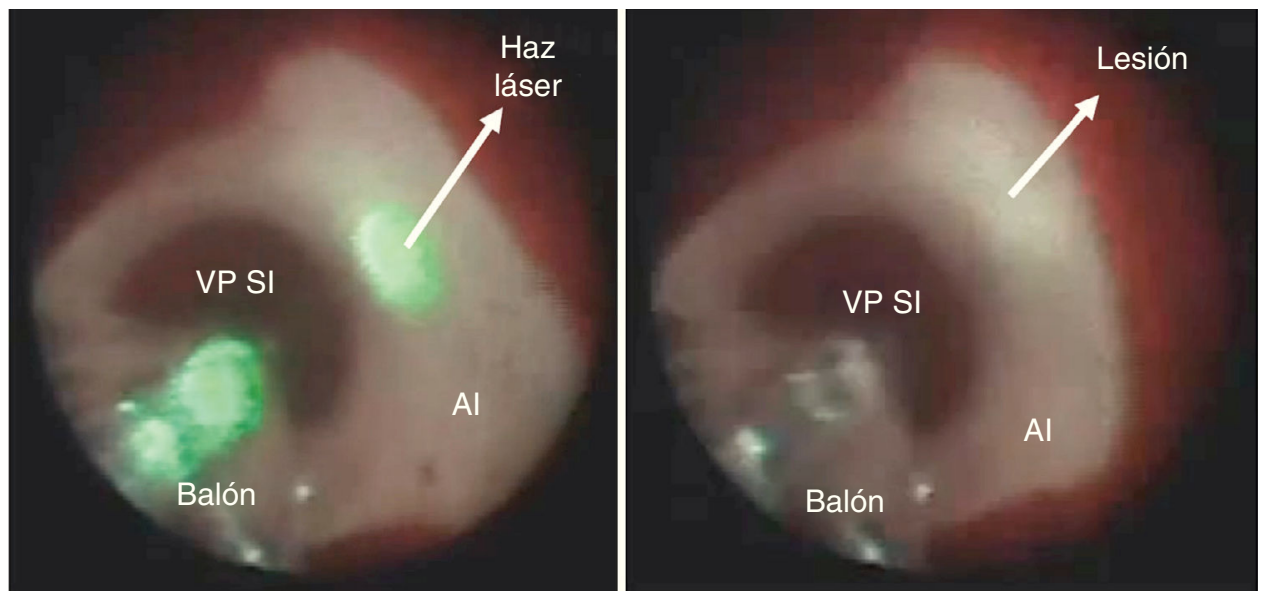

Figura 3 Balón en la vena pulmonar superior izquierda (VPSI), aplicando energía láser y aspecto de las lesiones causadas.

fuente de luz blanca y, d) otra de energía láser. El balón es de material transparente, distensible, con diámetro graduable en un rango de 9 a $35 \mathrm{~mm}$, característica que permite su adaptación a los diferentes tamaños del ostium de las venas pulmonares. El catéter es introducido a la aurícula izquierda mediante una camisa o sistema deflectable $15 \mathrm{Fr}$ y se conecta a una consola (CardioFocus ${ }^{\circledR}$ ) que dispone de tres puertos separados para el endoscopio, la fuente de luz y de energía láser. La luz blanca que emite el catéter permite el uso del endoscopio para visualizar el endocardio de la aurícula y guiar el catéter hacia el ostium de la vena pulmonar seleccionada. Una vez inflado el balón con $\mathrm{D}_{2} \mathrm{O}$ (agua pesada), se posiciona firmemente y se ajusta en tamaño en relación con el diámetro de la vena pulmonar; la energía láser es liberada durante 20 o 30 segundos, con una potencia en rango de 5,5 a 12 watts, sobre un segmento de aproximadamente 30 grados del ostium, desplazando el rayo de energía de manera secuencial y traslapada hasta abarcar la totalidad del circulo (figs. 3 y 4). En aquellas zonas en las que el contacto del balón láser no es adecuado, pueden administrarse lesiones complementarias de 5,5 Watts por 30 segundos ${ }^{38,39}$. Típicamente, el mayor poder de energía es aplicado a la porción anterior de las venas pulmonares dada su característica anatómica de mayor grosor del tejido y la menor potencia a los segmentos posteriores más delgados y relacionados con estructuras como el esófago. Metzner et al. y Bordignon et al. ${ }^{40-42}$ evaluaron por separado la cantidad de energía láser a utilizar con base en la región de interés y determinaron que el uso de al menos 8,5 watts en la pared posterior y hasta 10 watts en la anterior estuvieron relacionados con la mayor eficacia, validándose así la estrategia puramente guiada por endoscopio y reduciendo la necesidad de remapeo y ablación por radiofrecuencia punto a punto. Las consideraciones anatómicas de forma, dirección, tamaño y distancia de los ostium de las venas pulmonares reviste importancia adicional en la selección de la técnica con balón láser pues con frecuencia las venas pulmonares derechas no pueden aislarse como un todo dada la imposibilidad de visualizarlas em forma simultánea, en tanto que las izquierdas pueden abordarse mediante una circunferencia amplia o de manera individual. Wissner et al. ${ }^{43}$ informaron que la mayor eficacia en el asilamiento eléctrico de las

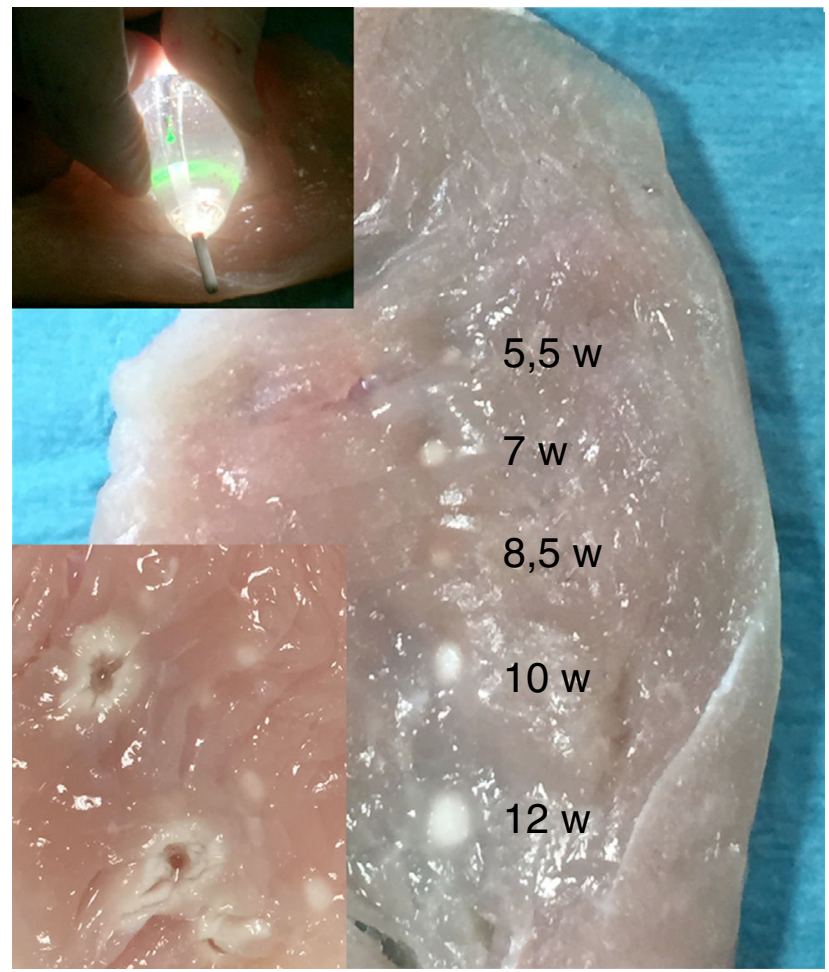

Figura 4 Experimento sobre músculo animal aplicando energía láser con balón (parte superior); tamaño de las lesiones producidas utilizando distintas potencias desde 5,5 a 12 watts (parte media derecha) y comparación con las lesiones causadas mediante energía de radiofrecuencia a 30 watts con un catéter de punta irrigada durante 30 segundos (recuadro inferior izquierdo).

venas pulmonares se logra cuando el abordaje es individual y no englobando las dos venas ipsilaterales, estrategia que estuvo más relacionada con la necesidad de "retocar" la ablación mediante radiofrecuencia punto a punto. Por su parte, Tsyganov et al. ${ }^{39}$ reportaron que las venas pulmonares superiores izquierdas de gran tamaño y las inferiores con ostium de aspecto ovalado, tienen menor porcentaje 
de éxito a largo plazo (un año). Dukkipathi et al. publicaron inicialmente en 2013 la experiencia multicéntrica con el uso del balón laser en los primeros 200 pacientes $^{44}$ e informaron una tasa de complicaciones baja, principalmente parálisis del nervio frénico en $2,5 \%$ y taponamiento cardiaco en $2 \%$. No hubo informe de fístula atrio-esofágica, estenosis significativa de las venas pulmonares, ictus o accidente cerebro-vascular. Más recientemente (septiembre de 2015) ${ }^{45}$ el mismo autor publicó la experiencia del estudio norteamericano de factibilidad del uso del sistema balón láser con la participación de diez centros en ese país. En contraste con la baja incidencia de complicaciones inicialmente informada, en este último estudio las complicaciones o eventos adversos primarios fueron del orden del $16,3 \%$, siendo la pericarditis el evento $\mathrm{m}\{$ as frecuente en $8,1 \%$, seguida de parálisis del nervio frénico en $5,8 \%$, taponamiento cardiaco en $3,5 \%$ e incluso muerte $(1,2 \%)$ sobre un total de 86 pacientes tratados. Los autores concluyen que el perfil de seguridad es razonable y la eficacia para realizar aislamiento eléctrico de las venas pulmonares es similar a la ablación por radiofrecuencia incluso cuando el balón láser manipulan operadores que hacen sus primeros casos. Estos resultados contrastan con la incidencia de complicaciones observada en la curva de aprendizaje de un solo centro europeo con reporte de parálisis del nervio frénico en sólo $2 \%$ de 150 pacientes, taponamiento e isquemia cerebral transitoria en $0,6 \%$ cada uno y $4 \%$ de lesión vascular o hematoma femoral ${ }^{46}$, para un total de $7 \%$ de complicaciones. Es posible que la adición del evento adverso primario "pericarditis" explique la alta tasa de complicaciones en los pacientes reportados por Dukipatti, pues eliminándola del total de complicaciones, la incidencia puede ser similar $(8,3 \%)$; sin embargo, la parálisis del nervio frénico todavía puede considerarse el doble de la observada en el estudio alemán de Perrota et al. ${ }^{46}$. Las lesiones térmicas causadas por el balón láser en el esófago han sido documentadas en un porcentaje similar al del uso de la ablación por radiofrecuencia irrigada convencional (18 vs. $15 \%$ ), pero la calidad de tales lesiones difiere en la mayor incidencia de ulceraciones hasta en un $57 \%$ de aquellos tratados con láser vs. ninguna úlcera en el grupo de radiofrecuencia ${ }^{47}$. En relación con el potencial embolígeno al sistema nervioso central, se han reportado eventos de isquemia cerebral silenciosa en el $11,4 \%$, cifra estadísticamente similar a la tasa reportada con radiofrecuencia convencional en rango de $7,4 \%$ a $18,2 \% 48$. El éxito agudo para alcanzar la desconexión de las venas pulmonares con la técnica endoscópica ha sido tan superlativa como del 98 al $100 \%$ con $86 \%$ de dicho aislamiento documentado hasta tres meses después del procedimiento ${ }^{49}$. No obstante, la tasa de éxito reportada a un año, tras una o dos intervenciones y medida en estado libre de medicación antiarrítmica, es del 63 y del 60,2\% respectivamente (Metzner-Dukkipati). Recientemente, el estudio prospectivo aleatorizado "Heart Light Study Investigators" comparó los resultados clínicos de la estrategia de balón láser con la ablación por radiofrecuencia irrigada punto a punto y encontró igual eficacia (>95\%) para lograr el aislamiento eléctrico de las venas pulmonares, tasa similar de eventos adversos (diferentes a la cardioversión) (5,9 vs. 6,4\%), mayor tiempo total y de fluoroscopia con la primera técnica pero similares tasas de recurrencia a un año $(40 \%)^{50}$.

\section{Conclusiones}

A pesar del creciente interés en la simplificación del procedimiento de aislamiento eléctrico de las venas pulmonares con la introducción de nuevas tecnologías como los catéteres multielectrodo y el balón láser, no se ha logrado popularizar su uso y los resultados de los estudios pioneros no fueron mejores y en algunos casos exhibieron un perfil de eficacia más bajo (caso de la tecnología MESH) que la estrategia de ablación con radiofrecuencia convencional punto a punto o con otra técnica emergente como el criobalón. El registro piloto europeo de ablación de fibrilación auricular publicado en diciembre de 2015, anota en una de sus tablas que en promedio $4,4 \%$ de los centros utilizaron catéter multielectrodo y un $0,8 \%$ técnica de láser balón. En relación con la práctica de la ablación de la fibrilación auricular persistente mediante radiofrecuencia en Europa, se informó que ninguno de los centros médicos que reportaron sus estadísticas, utilizaron las nuevas tecnologías aquí descritas. Los resultados de eficacia y seguridad para la tecnología multielectrodo son similares a los de la ablación por radiofrecuencia irrigada punto a punto, con discreto favor en relación a la reducción del tiempo total del procedimiento, de radiofrecuencia y de fluoroscopia. Sin embargo, algunas complicaciones parecen más frecuentes con el advenimiento de la ablación multipunto, tales como la parálisis del nervio frénico y la estenosis de las venas pulmonares, sin mencionar el incremento de la fístula cardio-esofágica que obligó al retiro del catéter multielectrodo $n M A R Q$ de Biosense. La incidencia también elevada de eventos isquémicos cerebrales silenciosos, parece tener una tendencia a corregirse con el ajuste de los protocolos de anticoagulación y con la desactivación de uno de los pares distal o proximal del catéter multielectrodos. De otra parte, el uso del oro en el nuevo diseño del PVAC es prometedor como estrategia que busca la reducción de la embolia cerebral silenciosa o sintomática pero a la fecha no hay estudios con suficiente poder para asegurar tal tendencia. Otro aspecto que se encuentra en la revisión de la literatura, es la necesidad no infrecuente de complementar los procedimientos de ablación multielectrodo con la tradicional ablación irrigada punto a punto, dada la persistencia de conexión eléctrica tras el primer paso de la ablación multipunto. Al parecer este hecho se debe al contacto no uniforme con la superficie endocárdica de los múltiples polos, fenómeno que parece tener relación con la alta variación anatómica de las venas pulmonares. El nuevo PVAC Gold con el diseño de 20 grados de inclinación (tilt) parece darle una mejoría en el contacto uniforme. Las recurrencias de fibrilación auricular tras una primera ablación con multielectrodo son similares a la radiofrecuencia irrigada punto a punto y este aspecto no parece haber alcanzado el ideal teórico de una transmuralidad más duradera. De hecho, es común que la ablación multielectrodo también requiera de un segundo procedimiento para alcanzar una eficacia tan aceptable como la alcanzada con la técnica tradicional. En relación con el balón láser, su eficacia y seguridad parecen similares a otras tecnologías como el criobalón, el PVAC y la radiofrecuencia irrigada convencional. Algunos artículos sugieren una curva de aprendizaje más corta que la requerida para radiofrecuencia irrigada de tan solo 15 casos, pero no más de 50 . La visualización 
endocárdica es muy interesante y la posibilidad de dirigir el rayo láser a una región bien definida bajo visión directa, da una percepción de mayor seguridad. Las complicaciones, sin embargo no se han reducido con el uso de esta nueva técnica; la incidencia de fístula cardio-esofágica es desconocida (no se ha reportado ningún caso), pero aunque inicialmente se informó como infrecuente, la parálisis del nervio frénico ha superado la incidencia causada por la radiofrecuencia irrigada tradicional. Llamativamente, se informa de una alta incidencia de pericarditis como su principal complicación, condición prácticamente ignorada en otras técnicas, series de casos, estudios multicéntricos o aleatorizados. Es posible que la mayor transmuralidad alcanzada con esta energía esté causando una complicación que será más reportada en el futuro cercano.

Los ideales teóricos de las nuevas tecnologías no han logrado alcanzarse y tan solo representan un paso más hacia su búsqueda insaciable. La complejidad de los procedimientos sigue siendo alta aunque alcanzar su accesibilidad y lograr su dominio promete ser menos complicado; si bien el tiempo total del procedimiento y de exposición radiológica parece estar en la tendencia a su reducción, sigue siendo de importante consideración; la seguridad o la incidencia de complicaciones no se ha reducido significativamente y no es seguro que se puedan incrementar en el futuro y la eficacia de las lesiones transmurales aunque prometedora no ha logrado redundar en mejores resultados o eficacia del tratamiento a largo plazo.

\section{Conflicto de intereses}

Los autores declaran no tener ningún conflicto de intereses.

\section{Bibliografía}

1. Haïssaguerre M, Gencel L, Fischer B, et al. Succesful catheter ablation of atrial fibrillation. J Cardiovasc Electrophysiol. 1994:1045-52.

2. Haïssaguerre $M^{1}$, Jaïs $P$, Shah DC, et al. Electrophysiological end point for catheter ablation of atrial fibrillation initiated from multiple pulmonary venous foci. Circulation. 2000;101:1409-17.

3. Haïssaguerre $M^{1}$, Jaïs $P$, Shah DC, et al. Spontaneous initiation of atrial fibrillation by ectopic beats originating in the pulmonary veins. New Eng J Med. 1998;339:659-66.

4. Scheiman M, Morady F. Nonpharmacological approaches to atrial fibrillation. Circulation. 2001;103:2120-30.

5. Chen SA, Hsieh MH, Tai CT, et al. Initiation of atrial fibrillation by ectopic beats originating from the pulmonary veins: electrophysiological characteristics, pharmacological responses and effects of radiofrequency ablation. Circulation. 1999;100:1879-86.

6. Buch E, Shivkumar K. Catheter ablation of atrial fibrillation. Advent of Second Generation Technologies. J Am Coll Cardiol. 2015;66:1361-3.

7. Mansour M, Forleo G, Pappalardo A, et al. Initial experience with the MESH catheter for pulmonary vein isolation in patients with paroxysmal atrial fibrillation. Heart Rhythm. 2008;5:1510-6.

8. De Greef Y, Tavernier R, Duytschaever M, Stockman D. Pulmonary vein isolation with the 30 and $35 \mathrm{~mm}$ high density mesh ablator. Europace. 2010;12:1428-34.
9. Koch L, Haeusler KG, Herm J, et al. Mesh ablator vs. cryoballoon pulmonary vein ablation of symptomatic paroxismal atrial fibrillation: results of the MACPAF study. Europace. 2012;14:1441-9.

10. Brunelli M, Raffa S, Große A, et al. Influence of the anatomic characteristics of the pulmonary vein ostium, the learning curve, and the use of a steerable sheath on success of pulmonary vein isolation with a novel multielectrode ablation catheter. Europace. 2012;14:331-40.

11. Mönnig G, Eckardt L. Multielectrode pulmonary vein ablation catheter. Current data on results and risks. Herzschr Elektrophys. 2014;25:236-40.

12. Laish-Farkash A, Khalameizer V, Fishman E, et al. Safety, efficacy, and clinical applicability of pulmonary vein isolation with circular multi-electrode ablation systems: PVAC vs. nMARQ for atrial fibrillation ablation. Europace. 2016;18:807-14.

13. Duytschaever M, Anne W, Apiashvili G, et al. Mapping and isolation of the pulmonary veins using the PVAC catheter. PACE. 2010;33:168-78.

14. De Greef Y, Buysschaert I, Schwagten B, et al. Duty-cycled multielectrode radiofrequency vs. conventional irrigated point-bypoint radiofrequency ablation for recurrent atrial fibrillation: comparative 3-year data. Europace. 2014;16:820-5.

15. Bulava A, Hanis J, Sitek D. Catheter ablation for paroxysmal atrial fibrillation: a randomized comparison between multielectrode catheter and point-by-point ablation. Pacing Clin Electrophysiol. 2010;33:1039-46.

16. Bittner A, Mönnig G, Zellerhoff S, et al. Randomized study comparing duty-cycled bipolar and unipolar radiofrequency with point-by-point ablation in pulmonary vein isolation. Heart Rhythm. 2011;8:1383-90.

17. Boersma LV, Wijffels MC, Oral H, et al. Pulmonary vein isolation by duty-cycled bipolar and unipolar radiofrequency energy with a multielectrode ablation catheter. Heart Rhythm. 2008;5:1635-42.

18. Wieczorek M, Hoeltgen R, Brueck M, et al. Pulmonary vein isolation by duty-cycled bipolar and unipolar antrum ablation using a novel multielectrode ablation catheter system: first clinical results. J Interv Card Electrophysiol. 2010;27:23-31.

19. Buist TJ, Gal P, Ottervanger JP, et al. Association between pulmonary vein orientation and ablation outcome in patients undergoing multi-electrode ablation for atrial fibrillation. J Cardiovasc Comput Tomogr. 2016 May-Jun;10:251-7.

20. Mulder AAW, Wijffels MCEF, Wever EFD, Boersma LVA. Freedom from paroxysmal atrial fibrillation after successful pulmonary vein isolation with pulmonary vein ablation catheter-phased radiofrequency energy: 2-year follow-up and predictors of failure. Europace. 2012;14:818-25.

21. De Greef Y, Buysschaert I, Schwagten B, et al. Duty-cycled multielectrode radiofrequency vs. conventional irrigated point-bypoint radiofrequency ablation for recurrent atrial fibrillation: comparative 3-year data. Europace. 2014;16:820-5.

22. Hummel J, Michaud G, Hoyt R, et al. Phased RF ablation in persistent atrial fibrillation. Heart Rhythm. 2014;11:202-9.

23. Raffa S, GroBe A, Brunelli M, et al. Voltage mapping and pacing to assess the level of pulmonary venous isolation achieved with a novel circular multielectrode ablation catheter. Europace. 2010;12:933-40.

24. Reddy VY, Neuzil P, dÁvila A, et al. Ballon catheter ablation to treat paroxismal atrial fibrillation: What is the level of pulmonary venous isolation? Heart Rhythm. 2008;5:353-60.

25. Malmborg H, Christersson C, Lonnerholm S, et al. Comparison of effects on coagulation and inflammatory markers using a duty-cycled bipolar and unipolar radiofrequency pulmonary vein ablation catheter vs. a cryoballoon catheter for pulmonary vein isolation. Europace. 2013;15:798-804.

26. Casella M, Dello Russo MA, Russo E, et al. Biomarkers of myocardial injury with differen tenergy sources for atrial fibrillation ablation. Cardiology J. 2014;5:516-23. 
27. von Bary C, Dornia C, Kirchner G, et al. Esophageal tissue injury following pulmonary vein isolation using the PVAC: assessment by endoscopy and magnetic resonance imaging. Pacing $\mathrm{Clin}$ Electrophysiol. 2013;36:477-85.

28. Ahsan SY, Flett AS, Lambiase PD, Segal OR. First report of phrenic nerve injury during pulmonary vein isolation using the ablation frontiers pulmonary vein ablation catheter. J Interv Card Electrophysiol. 2010;29:187-90.

29. Gaita F, Leclercq JF, Schumacher B, et al. Incidence of silent cerebral thromboembolic lesions after atrial fibrillation ablation may change according to technology used: comparison of irrigated radiofrequency, multipolar nonirrigated catheter and cryoballoon. J Cardiovasc Electrophysiol. 2011;22:961-8.

30. Verma A, Debruyne P, Nardi S, Deneke T, Degreef Y, et al. Evaluation and reduction of asymptomatic cerebral embolism in ablation of atrial fibrillation, but high prevalence of chronic silent infarction: results of the ERACE trial. Circ Arrhythm Electrophysiol. 2013;6:835-42.

31. Dong J, Vasamreddy CR, Jayam V, et al. Incidence and predictors of pulmonary vein stenosis following catheter ablation of atrial fibrillation using the anatomic pulmonary vein ablation approach: results from paired magnetic resonance imaging. J Cardiovasc Electrophysiol. 2005;16:845-52.

32. Compier MG, Leong DP, Marsan NA, et al. Duty-cycled bipolar/unipolar radiofrequency ablation for symptomatic atrial fibrillation induces significant pulmonary vein narrowing at longterm follow-up. Europace. 2013;15:690-6.

33. De Greef $Y$, Dekker L, Boerma L, et al. Low rate of asymptomatic cerebral embolism and improved procedural efficiency with the novel pulmonary vein ablation catheter GOLD: results of the PRECISION GOLD trial. Europace. 2016;18:687-95.

34. Richter B, Gwechenberger M, Kriegisch M, et al. Combined circular multielectrode catheter and point-by-point ablation is superior to point-by-point ablation alone in eliminating atrial fibrillation. International Journal of Cardiology. 2013;168:3721-7.

35. Choo WK, Farwell D, Harris S. Experience of atrial fibrillation ablation in a new cardiac centre using three-dimensional mapping and multielectrode duty-cycled radiofrequency ablation. Archives of Cardiovascular Disease. 2011;104:396-402.

36. Burri $\mathrm{H}$, Park $\mathrm{Cl}$, Poku N, et al. Pulmonary Vein Isolation for Paroxysmal Atrial Fibrillation Using a Circular Multipolar Ablation Catheter. Safety and Efficacy Using Low- Power Settings. J Cardiovasc Electrophysiol. 2016;27:170-4.

37. Wakili R, Siebermair J, Fichtner S, et al. One year clinical outcome after ablation with a novel multipolar irrigated ablation catheter for treatment of atrial fibrillation: potential implications for clinical use. Europace. 2016;18:1170-8.

38. Bordignon S, Chun KR, Gunawardene M, et al. Energy titration strategies with the endoscopic ablation system: lessons from the high-dose vs. low-dose laser ablation study. Europace. 2013;15:685-9.

39. Tsyganov A, Petru J, Skoda J, et al. Anatomical predictors for successful pulmonary vein isolation using balloon-based technologies in atrial fibrillation. J Interv Card Electrophysiol. 2015;44:265-71.

40. Metzner A, Wissner E, Lin T, et al. Ballon devices for atrial fibrillation therapy. Arrhythmia \& Electrophysiology Review. 2015;4:58-61.

41. Metzner A, Wissner E, Schoonderwoerd B. The influence of varying energy settings on efficacy and safety of endoscopic pulmonary vein isolation. Heart Rhythm. 2012;9:1380-5.

42. Bordignon S, Chun KR, Gunawardene M. Energy titration strategies with the endoscopic ablation system: lessons from the high-dose vs. low-dose laser ablation study. Europace. 2013;15:685-9.

43. Wissner E, Metzner A, Reissmann B, et al. Wide circumferential versus individual isolation of pulmonary veins using the endoscopic ablation system. J Cardiovasc Electrophysiol. 2014;25:253-8.

44. Dukkipati SR, Kuck KH, Neuzil P, et al. Pulmonary vein isolation using a visually guided laser balloon catheter: the first 200-patient multicenter clinical experience. Circ Arrhythm Electrophysiol. 2013;6:467-72.

45. Dukkipati SR, Cuoco F, Kutinsky I, et al. Pulmonary vein isolation using the visually guided laser balloon: a prospective, multicenter, and randomized comparison to standard radiofrequency ablation. J Am Coll Cardiol. 2015;66:1350-60.

46. Perrotta L, Bordignon S, Dugo D, et al. How to learn pulmonary vein isolation with a novel ablation device: learning curve effects using the endoscopic ablation system. J Cardiovasc Electrophysiol. 2014;25:1293-8.

47. Metzner A, Schmidt B, Fuernkranz A. Esophageal temperature change and esophageal thermal lesions after pulmonary vein isolation using the novel endoscopic ablation system. Heart Rhythm. 2011;8:815-20.

48. Herrera Siklody C, Deneke T, Hocini M, et al. Incidence of asymptomatic intracranial embolic events after pulmonary vein isolation: comparison of different atrial fibrillation ablation technologies in a multicenter study. J Am Coll Cardiol. 2011;58:681-8.

49. Dukkipati SR, Neuzil P, Kautzner J. The durability of pulmonary vein isolation using the visually guided laser balloon catheter: Multicenter results of pulmonary vein remapping studies. Heart Rhythm. 2012;9:919-25.

50. Dukkipati SR, Cuoco F, Kutinsky. For the HeartLight Study Investigators. Pulmonary vein isolation using the visually guided laser balloon: a prospective, multicenter, and randomized comparison to standard radiofrequency ablation. J Am Coll Cardiol. 2015;66:1350-60. 